\title{
Sugar Variation in Soybean Seed Assessed with a Rapid Extraction and Quantification Method
}

\author{
A. Hou, ${ }^{1,2}$ P. Chen, ${ }^{1}$ A. Shi, ${ }^{1,3}$ B. Zhang, ${ }^{1}$ and Y.-J. Wang ${ }^{4}$ \\ ${ }^{1}$ Department of Crop, Soil, and Environmental Sciences, University of Arkansas, Fayetteville, AR 72701, USA \\ ${ }^{2}$ Agriculture and Agri-Food Canada, Unit 100-101 Route 100, Morden, MB, Canada R6M 1 Y5 \\ ${ }^{3}$ Institute of Genomic Diversity, Cornell University, 175 Biotechnology Building, Ithaca, NY 14853, USA \\ ${ }^{4}$ Department of Food Science, University of Arkansas, Fayetteville, AR 72704, USA
}

Correspondence should be addressed to P. Chen, pchen@uark.edu

Received 23 April 2008; Accepted 16 June 2008

Recommended by Rodomiro Ortiz

\begin{abstract}
Sugar content in soybean [Glycine max (L) Merr.] seed is an important quality attribute for soyfood and feed. Rapid extraction and quantification of soluble sugars in soybean seed are essential for large-scale breeding selections. In this study, using water as extractant combined with a high-performance anion exchange chromatography with pulsed amperometric detection (HPAECPAD) system, variability and repeatability of sugar content were tested in 20 diverse soybean genotypes. Individual sugars were clearly resolved and identified, including sucrose, stachyose, raffinose, glucose, fructose, and verbascose. Analysis of variance (ANOVA) revealed a highly reproducible estimation of sucrose, stachyose, and raffinose. PI 243545 was confirmed as a unique germplasm with the highest sucrose $(105.48 \mathrm{mg} / \mathrm{g})$ and total sugar $(148.76 \mathrm{mg} / \mathrm{g})$ content. Two low-oligosaccharide accessions, PI 200508 and 03CB-14, were confirmed with extremely low concentrations of raffinose and stachyose. PI 417559 was identified as a high glucose and fructose germplasm. The identified germplasm with unique sugar profiles will be valuable in breeding specialty soybeans for improved sugar content. The sugar testing method will facilitate the screening of seed sugar profiles in a large-scale soybean breeding program.
\end{abstract}

Copyright (C 2009 A. Hou et al. This is an open access article distributed under the Creative Commons Attribution License, which permits unrestricted use, distribution, and reproduction in any medium, provided the original work is properly cited.

\section{Introduction}

Soybean [Glycine max (L) Merr.] seed is one of the major food sources for human and livestock. In addition to its rich protein $(40 \%)$ and oil $(20 \%)$ content, soybean seed also contains approximately $33 \%$ carbohydrates, up to $16.6 \%$ of which are soluble sugars [1]. The major sugars present in soybean seed include glucose, fructose, sucrose, raffinose, and stachyose [2,3]. Sucrose makes up 41.3-67.5\%, raffinose $5.2-15.8 \%$, and stachyose $12.1-35.2 \%$ of the total soluble sugars in soybean seed [3]. Trace amounts of other sugars have also been reported such as pinitol, myo-inositol, verbascose, galactose, arabinose, and mannose [2, 4]. Sugars in soybean seed affect soyfood quality, digestibility, and nutritional values. Soyfood such as soymilk, tofu, and natto is considered as healthy diets, and consumption is highly recommended by nutritionists and medical doctors [5]. In tofu, soymilk, natto, and many other soyfood products, desirable sugars including glucose, fructose, and sucrose contribute to the favorable sweet taste and are ready-todigest, while raffinose and stachyose are indigestible and cause undesirable flatulence and diarrhea [6].

Soybean cultivars are bred and grown for different end uses, such as regular beans for high protein and oil extraction, low-phytate beans for animal feed, low-linolenic beans for special low-trans-fat oil, large-seeded for tofu and soy milk, and small-seeded for natto production. Seed sugars vary in different soybean genotypes $[1,7-9]$. The concentration of various sugars is also significantly affected by processing methods in soybean meals [10]. Significant association $(-0.81)$ was found between the total sugar amount and the sum of protein and oil content in soybean seed [11]. Hymowitz et al. [8] reported that the total sugar and oil content in soybean seed were positively correlated 
(0.26), but both were negatively associated with protein $(-0.19$ and -0.63 , resp.). The soyfood markets prefer soybeans with high-sucrose, low-raffinose and stachyose content. One efficient way to improve the soybean sugar composition and marketability is through conventional breeding and genetic enhancement. Soybean breeding for improved sugar content has received considerable attention in recent years. Soybean breeding populations have been constructed using low-oligosaccharide germplasm PI 200508 and evaluated for developing soybean cultivars with reduced raffinose and stachyose [12]. Quantitative trait loci (QTLs) have also been mapped with molecular markers for soybean sucrose and oligosaccharide content [13-15]. The identified molecular markers will facilitate the breeding process for soybean sugar improvement. However, soybean cultivars with improved sugar profiles have not been reported.

Rapid sugar extraction and quantification are essential for large-scale soybean breeding programs because selections need to be accomplished for a large number of plant populations in a short period of time. A rapid and reliable procedure is also necessary for genetic screening and gene mapping. There are several protocols reported and used for sugar extraction in some soybean research programs, however, those protocols are not standardized and their repeatability is yet to be validated. Generally, sugar extraction is prepared from defatted or whole soybean meals using either ethanol or water as the solvent $[8,9,16]$. Effective sugar extraction has been achieved using $50-80 \%$ ethanol as solvent, however, complete extraction usually requires heating and repeated extraction steps with extraction time varying from 30 minutes to 24 hours $[8,9,14]$. Recently, Giannoccaro et al. [16] investigated the effects of solvent type, temperature, extraction time, solvent-to-sample ratio, and sample size on soybean sugar extraction. In that particular study, complete sugar recovery was achieved by either three repeated extractions using $50-80 \%$ ethanol or a single extraction using water as solvent. Sugar recovery by aqueous extraction remained comparable when extraction temperature varied between 25 and $50^{\circ} \mathrm{C}$. Their results further concluded that aqueous extraction for whole soybean meals with sample size of $0.1 \mathrm{~g}$, solvent-to-sample ratio of $10: 1$, and extraction time of 15 minutes at $25^{\circ} \mathrm{C}$ achieved comparable sugar results to various ethanolic methods. However, only one cultivar Hutcheson was evaluated in that study, and variations due to different extractions and quantifications were not examined. The objective of this study was to compare the variations of sugar content among selected diverse soybean genotypes and different extractions, following the procedures described by Giannoccaro et al. [16] in order to find a rapid and reliable procedure suitable for sugar analysis in large-scale soybean breeding.

\section{Materials and Methods}

2.1. Plant Materials and Sugar Extraction. Twenty soybean genotypes (Table 1) were used for this study, and seeds were harvested from the field in 2005 at the University of Arkansas Agricultural Experiment Station in Fayetteville, Ark, USA. These 20 genotypes were selected with various unique agronomic traits including six regular cultivars and germplasms (5002T, Jack, Ozark, PI 504510, PI 323556, and PI 417559), four small-seeded natto beans (Camp, SS516, V97-3000, and MFS-591), two large-seeded tofu beans (MFL-159 and MFL-552), one high protein line (R95-1705), one low phytate line (CX1834-1-2), one high total sugar line (PI 243545), and two low oligosaccharide lines (03CB-14 and PI 200508). Two accessions of wild species, PI 326581 (G. soja) and PI 153292, (G. gracilis) were also included for comparisons among species. Fifteen grams of seed were taken from each genotype, and two duplicate extractions were prepared from each sample. For each extraction, two repeated quantifications were conducted for soluble sugar concentrations. Standard sugar melibiose (Sigma Chemical Co., St. Louis, Mo, USA) was added in each sample as internal standard. CB03-14 and Ozark were also used as checks in each extraction and quantification to monitor the testing consistency and accuracy.

Seed sample was ground using a SmartGrind coffee bean grinder (Applica Consumer Products Inc., Fla, USA) and screened through a $250 \mu \mathrm{m}$ standard testing sieve (VWR International, USA). Sugar extraction was conducted following the procedures optimized by Giannoccaro et al. [16]. Briefly, duplicates of $0.15 \mathrm{~g}$ samples were weighed and placed in $2 \mathrm{~mL}$ Eppendorf microcentrifuge tubes containing $1.5 \mathrm{~mL}$ distilled deionized water. The mixture was vortexed and incubated at room temperature in a horizontal shaker at $200 \mathrm{rpm}$ for 15 minutes. The suspension was then centrifuged at $13000 \mathrm{rpm}$ for 10 minutes, and $500 \mu \mathrm{L}$ of the clear supernatant was transferred into a new $1.5 \mathrm{~mL}$ microcentrifuge tube and purified with $700 \mu \mathrm{L} 95 \%$ acetonitrile at room temperature for 30 minutes, and then centrifuged at $13000 \mathrm{rpm}$ for 10 minutes. A volume of $200 \mu \mathrm{L}$ of the supernatant was transferred into a $1.5 \mathrm{~mL}$ microcentrifuge tube and dried at $95^{\circ} \mathrm{C}$ in a Dry Bath Incubator (Fisher Scientific, Pittsburgh, Pa, USA) for approximately $45 \mathrm{~min}$ utes. The residue was redissolved in $200 \mu \mathrm{L} 65 \%$ acetonitrile and filtered through a $0.2 \mu \mathrm{m}$ membrane disc filter (Pall Co., Mich, USA).

Seed moisture was determined to adjust the sugar content to a dry weight basis. Two grams of ground soybean flour for each genotype were weighed in a predried aluminum weighing dish and dried in an oven at $130^{\circ} \mathrm{C}$ for 1 hour. The moisture content was calculated as moisture $(\%)=100$ $\times[$ (weight before drying) - (weight after drying) $] /$ (weight before drying).

\subsection{High-Performance Liquid Chromatography (HPLC) Sys-} tem. A Dionex DX500 HPAEC-PAD system equipped with a GS50 pump, an ED40 pulsed amperometric detector, an AS40 automated sampler with a $25 \mu \mathrm{L}$ injection loop, and a chromeleon chromatography management system (Dionex, Sunnyvale, CA, USA) was used for sugar identification and quantification. An analytical CarboPac PA10 pellicular anion-exchange resin column $(250 \times 4 \mathrm{~mm})$ preceded by a CarboPac PA10 guard column $(50 \times 4 \mathrm{~mm})$ and an AminoTrap column $(30 \times 3 \mathrm{~mm})$ was used for sugar separation.

An aliquot of $24 \mu \mathrm{L}$ purified samples was diluted with $576 \mu \mathrm{L}$ distilled water prior to HPLC injection. Sugars were 
TABLE 1: List of soybean genotypes used in this study and detected mean sugar content.

\begin{tabular}{|c|c|c|c|c|c|c|c|c|}
\hline \multirow{2}{*}{ Genotype } & \multirow{2}{*}{ Species } & \multirow{2}{*}{ Types and uses } & \multicolumn{6}{|c|}{ Sugar content $(\mathrm{mg} / \mathrm{g})^{(\mathrm{a})}$} \\
\hline & & & Sucrose ${ }^{(b)}$ & Raffinose $^{(\mathrm{c})}$ & Stachyose $\mathrm{d}^{(\mathrm{d})}$ & Glucose $^{(e)}$ & Fructose $^{(\mathrm{f})}$ & Total sugar ${ }^{(\mathrm{g})}$ \\
\hline PI 243545 & G. $\max$ & High-total sugar & 105.48 & 14.23 & 27.85 & 0.95 & 0.25 & 148.76 \\
\hline 03CB-14 & G. $\max$ & Low oligosaccharide & 88.09 & 3.76 & 4.14 & 1.33 & 0.95 & 98.27 \\
\hline $5002 \mathrm{~T}$ & G. $\max$ & Regular cultivar & 77.02 & 12.68 & 39.95 & 1.55 & 0.33 & 131.53 \\
\hline Jack & G. $\max$ & Regular cultivar & 64.37 & 10.58 & 37.37 & 0.68 & 0.25 & 113.25 \\
\hline PI 504510 & G. $\max$ & Regular germplasm & 63.02 & 5.9 & 36.7 & 0.8 & 0.3 & 106.72 \\
\hline Ozark & G. $\max$ & Regular cultivar & 58.02 & 12.09 & 33.44 & 1.25 & 0.4 & 105.2 \\
\hline SS516 & G. $\max$ & Small-seeded & 56.87 & 7.1 & 44.98 & 1.18 & 0.25 & 110.13 \\
\hline CX1834-1-2 & G. $\max$ & Low phytic acid & 56.09 & 9.9 & 40.48 & 0.85 & 0.23 & 107.55 \\
\hline R95-1705 & G. $\max$ & High protein & 55.14 & 3.45 & 37.29 & 1.87 & 1.3 & 99.05 \\
\hline V97-3000 & G. $\max$ & Small-seeded & 53.94 & 6.48 & 43.68 & 1.48 & 0.73 & 106.31 \\
\hline MFS-591 & G. $\max$ & Small-seeded & 52.47 & 5.38 & 49.95 & 1.18 & 0.2 & 109.18 \\
\hline Camp & G. $\max$ & Small-seeded & 52.04 & 10.7 & 38.68 & 0.38 & 0.13 & 101.93 \\
\hline PI 323556 & G. $\max$ & Regular germplasm & 43.7 & 9.47 & 34.53 & 1.33 & 0.37 & 89.4 \\
\hline MFL-552 & G. $\max$ & Large-seeded & 43.56 & 15.5 & 39.5 & 1.58 & 0.25 & 100.39 \\
\hline S02-529 & G. $\max$ & Regular germplasm & 40.14 & 12.73 & 37.03 & 1.68 & 0.9 & 92.48 \\
\hline MFL-159 & G. $\max$ & Large-seeded & 31.68 & 19.73 & 36.88 & 1.63 & 0.13 & 90.05 \\
\hline PI 200508 & G. $\max$ & Low oligosaccharide & 30.71 & 0.85 & 5.15 & 0.3 & 0.2 & 37.21 \\
\hline PI 417559 & G. $\max$ & Regular germplasm & 29.82 & 5.93 & 24.5 & 16.8 & 12.27 & 89.32 \\
\hline PI 326581 & G. soja & Wild species & 22.14 & 4.05 & 30.1 & 1.83 & 1.0 & 59.12 \\
\hline PI 153292 & G. gracilis & Wild species & 61.01 & 7.85 & 41.7 & 1.45 & 0.23 & 112.24 \\
\hline
\end{tabular}

(a) Standard sugar melibiose was added in each sample as internal standard. External sugar standards at concentrations of 0.5, 1.0, 2.0, 5.0, 10.0, 15.0, and $20.0 \mu \mathrm{g} / 600 \mu \mathrm{L}$ were prepared for creating calibration curves for each of the major sugars. The concentration of each sugar was estimated by regression of concentration and absorption of individual standard sugars; ${ }^{(\mathrm{b})} \mathrm{LSD}_{0.05}=9.07 ;{ }^{(\mathrm{c})} \mathrm{LSD}_{0.05}=1.35 ;{ }^{(\mathrm{d})} \mathrm{LSD}_{0.05}=4.26{ }^{(\mathrm{e})} \mathrm{LSD}_{0.05}=0.46$; $^{(\mathrm{f})} \mathrm{LSD}_{0.05}=0.36$; (g) $\operatorname{LSD}_{0.05}=9.13$.

eluted with $90 \mathrm{mM} \mathrm{NaOH}$ at a flow rate of $1.0 \mathrm{~mL} / \mathrm{min}$ under isocratic conditions. The mobile phase $90 \mathrm{mM} \mathrm{NaOH}$ was prepared by diluting carbonate-free HPLC grade $50 \%$ (w/w) stock solution in distilled water, filtered with a $0.45 \mu \mathrm{m}$ membrane, and degassed with compressed nitrogen gas for 30 minutes prior to being loaded. The data acquisition for each sample was 30 minutes, and the analyses of 40 samples were generally completed in 20 hours.

2.3. Sugar Identification, Quantification, and Statistical Analysis. Nine standard sugars, including pinitol, myo-inositol, galactose, glucose, fructose, sucrose, raffinose, stachyose, and verbascose, were obtained from Sigma Chemical Co. Sugars in the extracts were identified by comparing their retention times to those of the standard sugars. Sugar standards at concentrations of $0.5,1.0,2.0,5.0,10.0,15.0$, and $20.0 \mu \mathrm{g} / 600 \mu \mathrm{L}$ were prepared for creating calibration curves for each of the major sugars, including glucose, fructose, sucrose, raffinose, and stachyose. The sugar concentration in soybean seed was expressed as mg per g of dry seed matter. Analysis of variance (ANOVA) was performed using the general linear models (Proc GLM) of SAS 9.1 (SAS Institute Inc., Cary, NC, USA). Means of individual sugars in different genotypes were compared and separated by SAS Waller grouping at $P=.05$. Correlation analysis among different sugar compositions was conducted using Excel CORREL function.

\section{Results and Discussion}

3.1. HPAEC-PAD Chromatogram. Soybean seed sugars were eluted in the sequence of pinitol/myo-inositol, glucose, fructose, sucrose, raffinose, stachyose, and verbascose based on their retention times (Figure 1). Because pinitol and myoinositol were eluted at the same retention time (2.3 minutes), they could not be distinguished from each other by the HPLC system conditions in this study. Galactose was not detectable in mature dry soybean seed. A group of small molecules present before 3.8 minutes were not identifiable by the standard sugars used in this study. The internal standard melibiose was eluted at 8.0 minutes. Verbascose was identified as a minor sugar with a retention time of 21.3 minutes, which has not been reported in previous research. Future quantification of verbascose is important and valuable, especially for vegetable soybean seed during seed development or storage, when verbascose concentration is relatively high. Sucrose and stachyose represented the major sugars in large quantities identified in soybean genotypes (Table 1). Significant difference was detected for all the soluble sugars among the 20 soybean genotypes. The genotypic differences for sucrose, stachyose, and total soluble sugar appeared larger than those for raffinose, glucose, and fructose. Highly consistent estimates for major sugars were achieved across different extractions and replications (Tables $2 \& 3)$. 


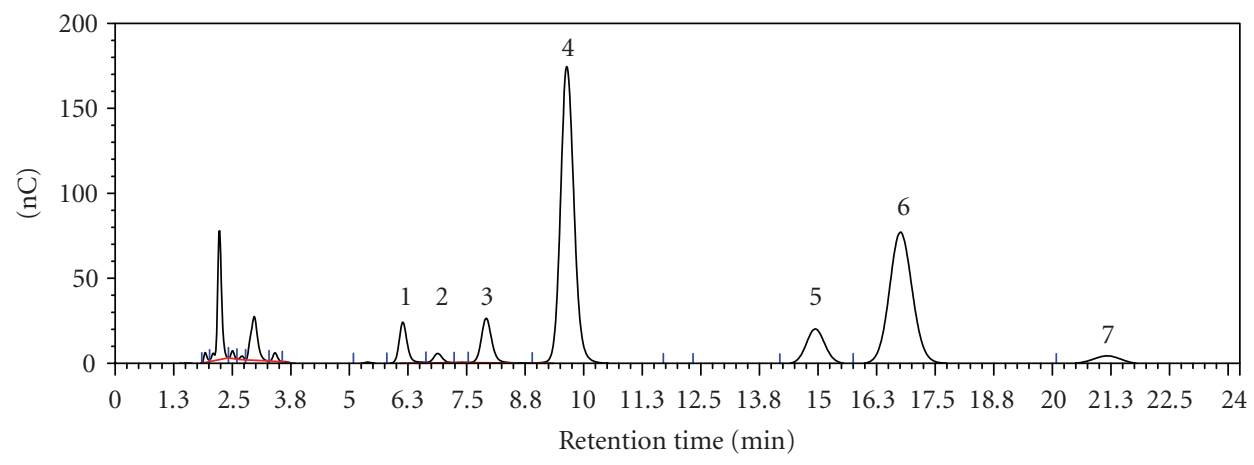

(a)

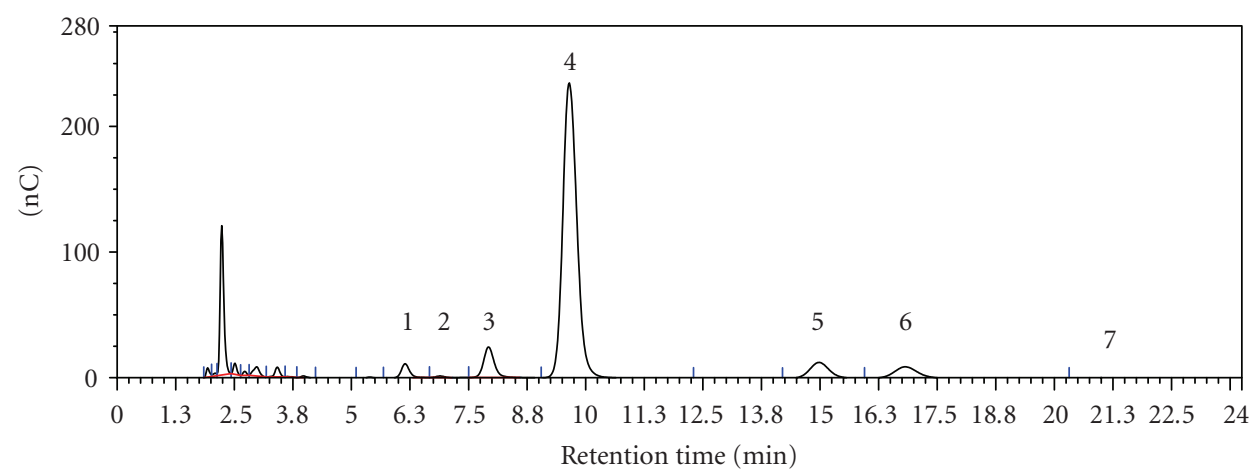

(b)

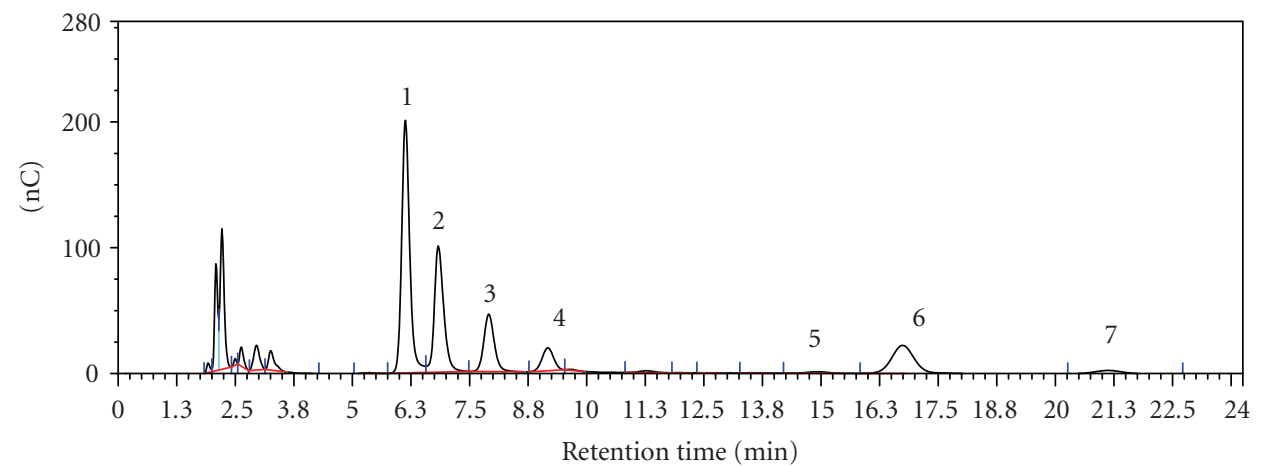

(c)

FIgURE 1: HPAEC-PAD chromotograms of soybean seed sugars in Ozark (a), 03CB-14 (b), and PI 417559 (c): glucose (1), fructose (2), melibiose (3), sucrose (4), raffinose (5), stachyose (6), and verbascose (7). Unidentified peaks are not labeled.

The use of water as extractant significantly shortened the extraction time when compared with using $80 \%$ ethanol as extractant $[8,9,16]$, therefore, that would allow for the screening of a large number of breeding lines and facilitate the development of soybean cultivars with desirable sugar profiles. Using water as extractant is also low cost associated with less labor input. The HPAEC-PAD detection method has been reported as sensitive, fast, and capable of separating individual soluble sugars in soybean seed [17]. The sensitivity and specificity of the present HPLC system enabled clear identification and consistent quantification of soybean sugars without much derivation as often observed in gas chromatography (GC) with flame ionization detection [8]. In previous reports using GC system, only a few major sugars, including sucrose, raffinose, and stachyose, were detected $[8,9]$. Identification of minor sugars such as glucose, fructose, and verbascose using methods in this research makes feasible the studies of these sugars in specialty soybean genotypes, and their changes during seed development and food processing. With the combination of rapid extraction and sensitive detection, following the procedures used in this study, the analyses of 40 samples can be completed in 24 hours.

3.2. Sucrose. Sucrose was eluted at the retention time of 9.6 minutes (Figure 1). The ANOVA results revealed significant variation for sucrose content among the 20 genotypes tested $(P<.0001)$, whereas no significant difference was detected 
between duplicate extractions $(P=.2284)$ (Table 2$)$. There were no significant interactions between genotype and extraction on sucrose content $(P=.2258)$. These results indicate that the extraction and quantification methods and conditions used in the present study are highly reproducible. Evidently, current methodology is very accurate in assessing sucrose content in given genotypes and, therefore, very efficient in ranking soybean genotypes for breeding selection.

Sucrose was the predominant soluble sugar, accounting for an average of $55.16 \%(33-90 \%)$ of the total sugar (Table 1). The average sucrose content was $53.45 \mathrm{mg} / \mathrm{g}$ among all G. $\max$ accessions screened, with more than three-fold differences between the extremes, ranging from 29.82 to $105.48 \mathrm{mg} / \mathrm{g}$. The highest sucrose content was detected in PI 243545 (105.48 mg/g, 79\% of total sugar), which was known as a large-seeded soybean germplasm with high total soluble sugar in our breeding program. The low-oligosaccharide breeding line, 03CB-14, also contained high sucrose $(88.09 \mathrm{mg} / \mathrm{g})$, accounting for $90 \%$ of its total sugar. However, another low-oligosaccharide line, PI 200508, only had $30.71 \mathrm{mg} / \mathrm{g}$ sucrose content. The two large- and four small-seeded food grade cultivars contained relatively low- (below average) sucrose content $(31.68-56.87 \mathrm{mg} / \mathrm{g}$ ). The regular commercial cultivars contained relatively high (above average) sucrose, whereas PI 417559 contained only $29.85 \mathrm{mg} / \mathrm{g}$ sucrose, accounting for $33 \%$ of its total sugar. The sucrose content in high-protein line, R95-1705 (55.14 mg/g), and low-phytate line, CX1834-1-2 (56.0 mg/g), was moderate. The lowest sucrose content was detected in the wild species G. soja PI 326581 (22.14 mg/g, 37.4\% of total sugar). In contrast, another wild species, G. gracilis PI 153292, had moderately high-sucrose content $(61.01 \mathrm{mg} / \mathrm{g})$, accounting for $54 \%$ of its total sugar.

Hymowitz and Collins [1] reported that the sucrose content in 195 diverse soybean genotypes ranged from 30 to $102 \mathrm{mg} / \mathrm{g}$, which is very similar to the sucrose variation observed in this study. The average sucrose content was $61 \mathrm{mg} / \mathrm{g}$, accounting for $57.5 \%$ of total soluble sugars on average. Hymowitz et al. [8] also showed that the sucrose content varied with soybean maturity groups (MG), ranging from 41 to $54 \mathrm{mg} / \mathrm{g}$ in MG 00 to IV, representing 55.4 to $62.8 \%$ of total sugars. More recently, Hartwig et al. [7] reported a mean sucrose content of 39.6 to $55.2 \mathrm{mg} / \mathrm{g}$ in multiple soybean cultivars evaluated over two years. The average sucrose content and its proportion to total sugars in 18 G. max accessions screened in this study were comparable to the values reported previously in other soybean genotypes $[1,7,16]$. The sucrose content of $105.48 \mathrm{mg} / \mathrm{g}$ in PI 243545 was similar to that of the highest sucrose genotypes identified by Hymowitz and Collins [1]. In contrast, low-sucrose content was observed previously in the wild species $G$. soja ( $24 \mathrm{mg} / \mathrm{g}$ on average) and G. gracilis (36 mg/g on average) [1]. In this study, the G. gracilis PI 153292 (61.01 mg/g) contained much higher sucrose than previously reported [1], whereas G. soja PI 326581 (22.14 mg/g) was similar to what was reported previously [1].

3.3. Stachyose. Stachyose was the second richest soluble sugar detected in soybean seed with a retention time of
16.8 minutes (Figure 1). The ANOVA indicated a significant variation among genotypes $(P<.0001)$, but not significant between extractions $(P=.9950)$ and genotype $\mathrm{x}$ extraction interactions $(P=.2227)$ (Table 2$)$. Therefore, the extraction method and HPLC conditions used in this study produced reliable stachyose results in differentiating and ranking genotypes for breeding selection.

Relatively high stachyose content was detected in most of G. max lines tested, excluding 03CB-15 and PI 200508, ranging from 24.5 to $45.75 \mathrm{mg} / \mathrm{g}$. High stachyose content was also detected in two wild species $(41.7 \mathrm{mg} / \mathrm{g}$ in $G$. gracilis and $30.1 \mathrm{mg} / \mathrm{g}$ in G. soja, resp.). The average stachyose content in $18 \mathrm{G}$. max soybeans was $31.97 \mathrm{mg} / \mathrm{g}$, and the average percentage of stachyose in total sugar was $33.97 \%$. The average content and percentage of stachyose in total sugar were similar to those in previous reports $[7,8$, 16]. However, the two low-oligosaccharide lines, 03CB14 and PI 200508, contained significantly lower stachyose content (4.14 and $5.15 \mathrm{mg} / \mathrm{g}$, resp.) than other genotypes, accounting for only 4.2 and $13.8 \%$ of their total sugars. The stachyose content observed in PI 200508 in this study was in good agreement with the previous report, in which breeding populations derived from PI 200508 contained low-stachyose content of less than $8.1 \mathrm{mg} / \mathrm{g}$ [12]. The highsucrose and low-stachyose content in 03CB-14 make it a valuable and unique germplasm for breeding specialty food grade soybeans (Figure $1(\mathrm{~b})$ ). In addition, the highsucrose line, PI 243545, and the low-sucrose germplasm, PI 417559, also contain relatively low stachyose $(27.85 \mathrm{mg} / \mathrm{g}$ and $24.5 \mathrm{mg} / \mathrm{g}$, resp.) and may be valuable for future genetic studies or breeding purposes. Stachyose constitutes the most of undesirable oligosaccharides in soybean seed. Reduction of stachyose improves food quality for human and other animals by increasing digestibility and metabolizing energy. The low-stachyose lines identified or confirmed in this study will benefit future food grade soybean breeding as unique germplasm resources.

3.4. Minor Sugars. Low concentration of minor sugars including glucose, fructose, raffinose, and verbascose was eluted at 6.2, 6.8, 9.6, 15, and 16.8 minutes, respectively (Figure 1). The average content of raffinose was $8.54 \mathrm{mg} / \mathrm{g}$ in 18 G. max soybeans studied (Table 1), accounting for $8.5 \%$ of total sugars. The lowest raffinose content $(0.85 \mathrm{mg} / \mathrm{g})$ was detected in PI 200508, confirming it as a low-oligosaccharide germplasm. Genetic variation for raffinose was significant $(P<.0001)$ among the 20 genotypes tested, with a range of $0.85-19.73 \mathrm{mg} / \mathrm{g}$. There was no significant variation for extractions and genotype $\mathrm{x}$ extraction interactions, indicating a reliable detection of this sugar and consistent sorting of the genotypes. Therefore, the extraction method and HPLC conditions used in this study are adequate for separating and ranking soybean genotypes in breeding selection.

The glucose and fructose contents were below $2 \mathrm{mg} / \mathrm{g}$ in all genotypes except PI 417559, which contained much higher concentration of glucose $(16.8 \mathrm{mg} / \mathrm{g})$ and fructose $(12.27 \mathrm{mg} / \mathrm{g})$ than other genotypes tested. This six- to eightfold increase in glucose and fructose may have resulted from a genetic mutation (Figure 1(c)). Therefore, PI 417559 
TABLE 2: Analyses of variance for five major sugar and total sugar contents in twenty soybean genotypes.

\begin{tabular}{|c|c|c|c|c|}
\hline Sugar/source ${ }^{(a)}$ & $\mathrm{DF}^{(\mathrm{b})}$ & Mean square & $F$ value & $\operatorname{Pr}>F$ \\
\hline \multicolumn{5}{|l|}{ Sucrose } \\
\hline Genotype & 19 & 1188.80 & 25.55 & $<.0001$ \\
\hline Extraction & 1 & 69.80 & 1.50 & .2284 \\
\hline Genotype $\mathrm{x}$ extraction & 19 & 61.69 & 1.33 & .2258 \\
\hline \multicolumn{5}{|l|}{ Stachyose } \\
\hline Genotype & 19 & 535.99 & 51.04 & $<.0001$ \\
\hline Extraction & 1 & 0.07 & 0.01 & .9950 \\
\hline Genotype $\mathrm{x}$ extraction & 19 & 13.98 & 1.33 & .2227 \\
\hline \multicolumn{5}{|l|}{ Raffinose } \\
\hline Genotype & 19 & 87.52 & 82.00 & $<.0001$ \\
\hline Extraction & 1 & 0.26 & 0.24 & .6265 \\
\hline Genotype x extraction & 19 & 1.56 & 1.46 & .1572 \\
\hline \multicolumn{5}{|l|}{ Glucose } \\
\hline Genotype & 19 & 37.58 & 303.28 & $<.0001$ \\
\hline Extraction & 1 & 1.72 & 13.88 & .0006 \\
\hline Genotype $\mathrm{x}$ extraction & 19 & 3.52 & 28.39 & $<.0001$ \\
\hline \multicolumn{5}{|l|}{ Fructose } \\
\hline Genotype & 19 & 21.67 & 290.03 & $<.0001$ \\
\hline Extraction & 1 & 0.53 & 7.11 & .0114 \\
\hline Genotype $\mathrm{x}$ extraction & 19 & 0.24 & 3.33 & .0009 \\
\hline \multicolumn{5}{|l|}{ Total sugar } \\
\hline Genotype & 19 & 2102.63 & 43.72 & $<.0001$ \\
\hline Extraction & 1 & 61.63 & 1.28 & .2649 \\
\hline Genotype $\mathrm{x}$ extraction & 19 & 3204.59 & 3.51 & .0005 \\
\hline
\end{tabular}

TABLE 3: Means of individual sugars in 20 soybean genotypes over replications of two duplicate extractions.

\begin{tabular}{|c|c|c|c|c|}
\hline \multirow{3}{*}{ Sugar } & \multicolumn{4}{|c|}{ Average sugar content $(\mathrm{mg} / \mathrm{g})^{(\mathrm{a})}$} \\
\hline & \multicolumn{2}{|c|}{ Extraction I } & \multicolumn{2}{|c|}{ Extraction II } \\
\hline & Replication 1 & Replication 2 & Replication 1 & Replication 2 \\
\hline Glucose & 0.18 & 0.17 & 0.13 & 0.12 \\
\hline Fructose & 0.12 & 0.12 & 0.11 & 0.05 \\
\hline Sucrose & 4.49 & 4.47 & 4.82 & 4.68 \\
\hline Raffinose & 0.86 & 0.91 & 0.91 & 0.96 \\
\hline Stachyose & 3.41 & 3.42 & 3.45 & 3.45 \\
\hline Total sugar & 9.05 & 9.09 & 9.35 & 9.12 \\
\hline
\end{tabular}

(a) Two duplicate extractions following Giannoccaro et al. [16] were prepared from each sample, and each extraction was analyzed in two replicates.

may serve as a valuable germplasm for genetic studies and breeding implications. The significant variation due to extractions and genotype $\mathrm{x}$ extraction interactions suggested a poor reproducibility in quantifications of glucose and fructose under conditions used in this study. This may be due to the fact that the concentration of these two sugars was very low and it is not feasible to determine the actual concentrations. However, in breeding efforts to improve glucose and fructose content, the current approach can be used to screen genetic materials with high glucose and fructose such as PI 417559. The high glucose and fructose germplasm will have extra values if incorporated in the highsucrose and low-oligosaccharide genetic background.

3.5. Total Sugar Content and Correlation Analysis. Significant variation existed in total soluble sugar content among all soybean genotypes tested $(P<.001$, Tables $1 \& 2)$, whereas the variation between extractions was not significant. The total sugar content among the 20 genotypes ranged from 37.21 (PI 200508) to 148.76 (PI 243545) $\mathrm{mg} / \mathrm{g}$, with a mean of $96.9 \mathrm{mg} / \mathrm{g}$. Different end-use soybean types contained similar levels of total sugars except PI 243545 being significantly 
high and PI 200580 and PI $326581(59.12 \mathrm{mg} / \mathrm{g})$ extremely low. The low-oligosaccharide line PI 200508 contained the least amount of total soluble sugar $(37.2 \mathrm{mg} / \mathrm{g})$ with $82.5 \%$ of which being sucrose. The total sugar content is primarily composed of sucrose and oligosaccharides (raffinose and stachyose). The glucose, fructose, or verbascose content in soybean seed has not been quantified in previous investigations due to the trace amount of these sugars $[1,8]$. Total sugar content in soybean seed ranged from 62 to $166 \mathrm{mg} / \mathrm{g}$ in previous reports $[1,8]$, which was very similar to the results obtained in this study. The total sugar amount previously reported for G. gracilis ranged from 48 to $99 \mathrm{mg} / \mathrm{g}$ [1], which was much lower than that observed in PI 153292 in this study $(112.24 \mathrm{mg} / \mathrm{g})$. However, the total sugar content in G. soja PI 326581 was in the range as reported for G. soja before [1].

Correlation analyses revealed that total sugar content was positively and significantly correlated with sucrose $(r=$ $\left.0.7930^{* *}\right)$, raffinose $\left(r=0.4827^{* *}\right)$, or stachyose $(r=$ $\left.0.4954^{* *}\right)$, respectively. Positive and significant correlations also existed between raffinose and stachyose $\left(r=0.3687^{* *}\right)$. These correlationships coincided well with previous results [8]. Additional analyses showed a strong positive correlation between glucose and fructose $\left(r=0.9796^{* *}\right)$, but a negative relationship between glucose and sucrose $\left(r=-0.3125^{* *}\right)$. A weak and negative correlation was also found between fructose and sucrose $\left(r=-0.2980^{*}\right)$, stachyose $(r=$ $\left.-0.2219^{*}\right)$, raffinose $(r=-0.2022)$, or total sugar $(r=$ $-0.1420)$. This negative correlationship is well illustrated by PI 417559, which had the highest glucose and fructose content but low-total sugar $(89.32 \mathrm{mg} / \mathrm{g})$ and sucrose $(29.82 \mathrm{mg} / \mathrm{g})$. The increase of glucose and fructose content was apparently at the cost of other sugars such as sucrose and oligosaccharides. Further investigation is needed to improve the digestible sugars (glucose, fructose, and sucrose), while reduce the oligosaccharides (raffinose and stachyose) using the unique germplasm lines such as 03CB-14 and PI 417559.

\section{Conclusions}

Significant variation was observed for individual and total sugar contents in 20 diverse soybean genotypes tested in this study. Reproducible estimates were achieved for sucrose, stachyose, raffinose, and total sugars with the HPLC system used. Due to the low concentrations of glucose and fructose in soybean seed, quantification of such sugars was variable and relatively inaccurate. However, it is possible to screen and select soybean germplasm such as PI 417559 with high glucose and fructose. Sucrose was the predominant sugar in soybean seed followed by stachyose. The highest sucrose $(105.48 \mathrm{mg} / \mathrm{g})$ and total sugar $(148.76 \mathrm{mg} / \mathrm{g})$ content was found in PI 243545, whereas the least sucrose and total sugar was found in wild species G. soja PI 326581 $(22.14 \mathrm{mg} / \mathrm{g})$ and PI $200508(37.21 \mathrm{mg} / \mathrm{g})$, respectively. Two low-oligosaccharide breeding lines, PI 200508 and 03CB14 , were confirmed with extremely low concentrations of raffinose and stachyose. The identified germplasm with unique sugar profiles is particularly valuable in breeding specialty soybeans for food and feed purposes. Total sugar content was positively correlated with sucrose, raffinose, and stahcyose content. Positive correlations were also found between raffinose and stachyose, and between glucose and fructose. Negative correlations existed between glucose and sucrose, fructose and sucrose, and fructose and stahcyose. Further investigations are needed to examine the environmental effects on sugar contents in the unique soybean germplasm identified in this study.

\section{Acknowledgment}

The funding for this research was partially provided by a USDA National Research Initiatives Grant (no. 2004-3550314114).

\section{References}

[1] T. Hymowitz and F. I. Collins, "Variability of sugar content in seed of Glycine $\max ($ L.) Merril and G. soja Sieb and Zucc," Agronomy Journal, vol. 66, no. 2, pp. 239-240, 1974.

[2] A. C. Eldridge, L. T. Black, and W. J. Wolf, "Carbohydrate composition of soybean flours, protein concentrates, and isolates," Journal of Agricultural and Food Chemistry, vol. 27, no. 4, pp. 799-802, 1979.

[3] B. Yazdi-Samadi, R. W. Rinne, and R. D. Seif, "Components of developing soybean seeds: oil, protein, sugars, starch, organic acids, and amino acids," Agronomy Journal, vol. 69, no. 3, pp. 481-486, 1977.

[4] T. F. Schweizer, I. Horman, and P. Wursch, "Low molecular weight carbohydrates from leguminous seeds, a new disaccharide, galactopinitol," Journal of the Science of Food and Agriculture, vol. 29, no. 2, pp. 148-154, 1978.

[5] M. J. Messina, "Emerging evidence on the role of soy in reducing prostate cancer risk," Nutrition Reviews, vol. 61, no. 4, pp. 117-131, 2003.

[6] J. J. Rackis, "Oligosaccharides of food legumes: alphagalactosidase activity and flatus problem," in Physilogical Effects of Food Carbohydrates, A. Jeanes and J. Hodge, Eds., pp. 207-222, American Chemical Society, Washington, DC, USA, 1975.

[7] E. E. Hartwig, T. M. Kuo, and M. M. Kenty, "Seed protein and its relationship to soluble sugars in soybean," Crop Science, vol. 37, no. 3, pp. 770-773, 1997.

[8] T. Hymowitz, F. I. Collins, J. Panczner, and W. M. Walker, "Relationship between the content of oil, protein, and sugar in soybean seed," Agronomy Journal, vol. 64, no. 5, pp. 613-616, 1972.

[9] T. M. Kuo, J. F. VanMiddlesworth, and W. J. Wolf, "Content of raffinose oligosaccharides and sucrose in various plant seeds," Journal of Agricultural and Food Chemistry, vol. 36, no. 1, pp. 32-36, 1988.

[10] C. M. Grieshop, C. T. Kadzere, G. M. Clapper, et al., "Chemical and nutritional characteristics of United States soybeans and soybean meals," Journal of Agricultural and Food Chemistry, vol. 51, no. 26, pp. 7684-7691, 2003.

[11] C. W. Geater and W. R. Fehr, "Association of total sugar content with other seed traits of diverse soybean cultivars," Crop Science, vol. 40, no. 6, pp. 1552-1555, 2000.

[12] J. D. Neus, W. R. Fehr, and S. R. Schnebly, "Agronomic and seed characteristics of soybean with reduced raffinose and stachyose," Crop Science, vol. 45, no. 2, pp. 589-592, 2005.

[13] C. D. Feng, M. Morsey, E. Giannoccaro, B. Zhang, and P. Chen, "Soybean seed sugar content and quantitative trait 
loci mapping," in Plant Nutrition for Food Security, Human Health and Environmental Protection, C. J. Li, Ed., pp. 438-439, Tsinghua University Press, Beijing, China, 2005.

[14] H.-K. Kim, S.-T. Kang, J.-H. Cho, M.-G. Choung, and D.-Y. Suh, "Quantitative trait loci associated with oligosaccharide and sucrose contents in soybean (Glycine max L.)," Journal of Plant Biology, vol. 48, no. 1, pp. 106-112, 2005.

[15] P. J. Maughan, M. A. Saghai Maroof, and G. R. Buss, "Identification of quantitative trait loci controlling sucrose content in soybean (Glycine max)," Molecular Breeding, vol. 6, no. 1, pp. 105-111, 2000.

[16] E. Giannoccaro, Y.-J. Wang, and P. Chen, "Effects of solvent, temperature, time, solvent-to-sample ratio, sample size, and defatting on the extraction of soluble sugars in soybean," Journal of Food Science, vol. 71, no. 1, pp. C59-C64, 2006.

[17] E. Giannoccaro, Y.-J. Wang, and P. Chen, "Comparison of two HPLC systems and an enzymatic method for quantification of soybean sugars," Food Chemistry, vol. 106, no. 1, pp. 324-330, 2008. 


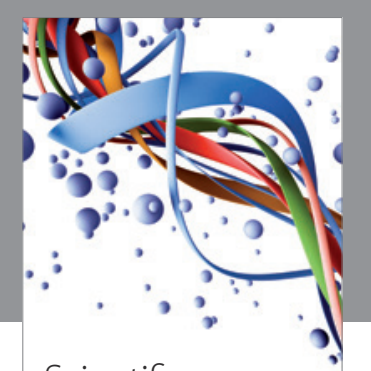

Scientifica
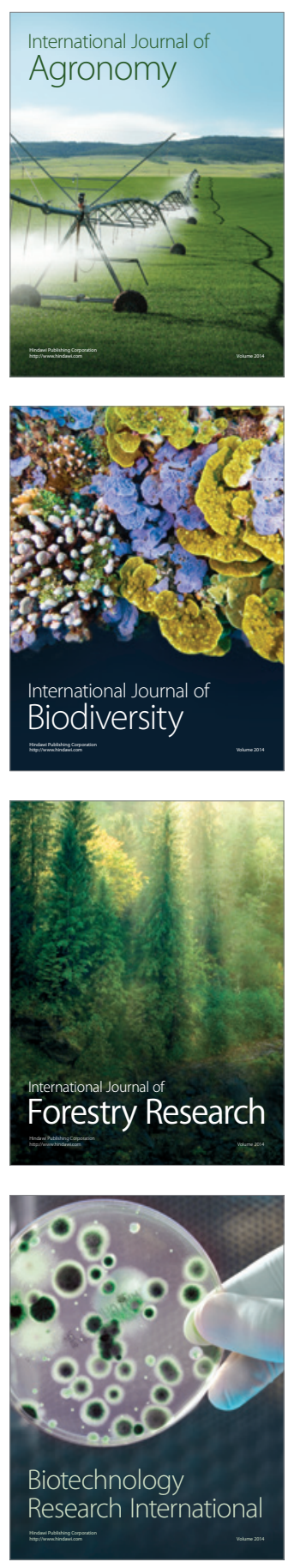
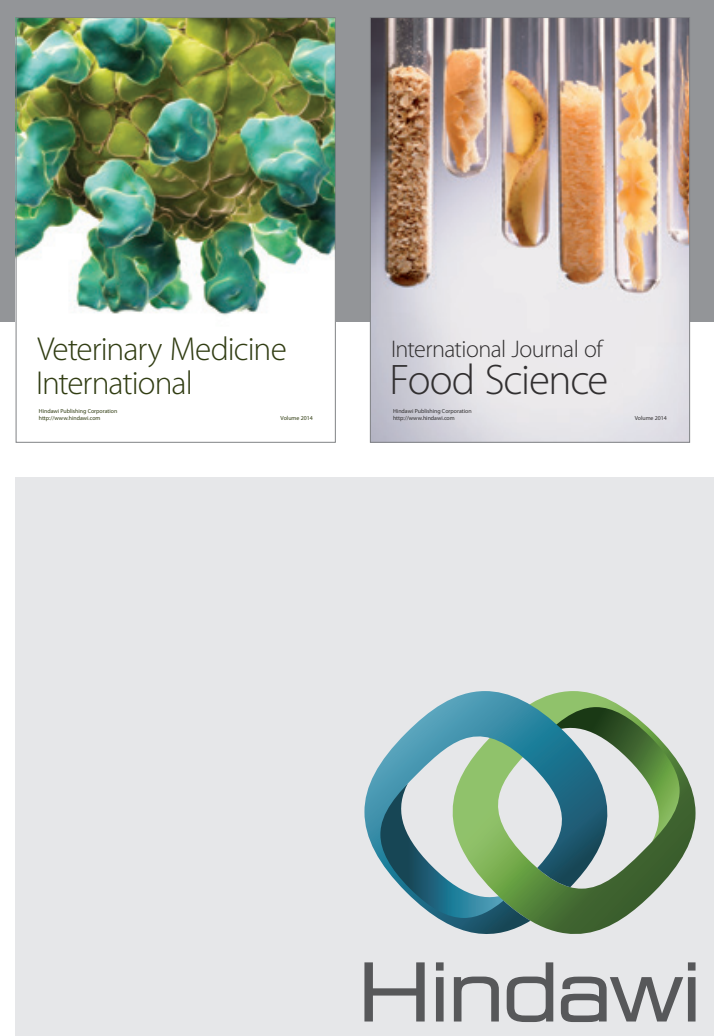

Submit your manuscripts at

http://www.hindawi.com
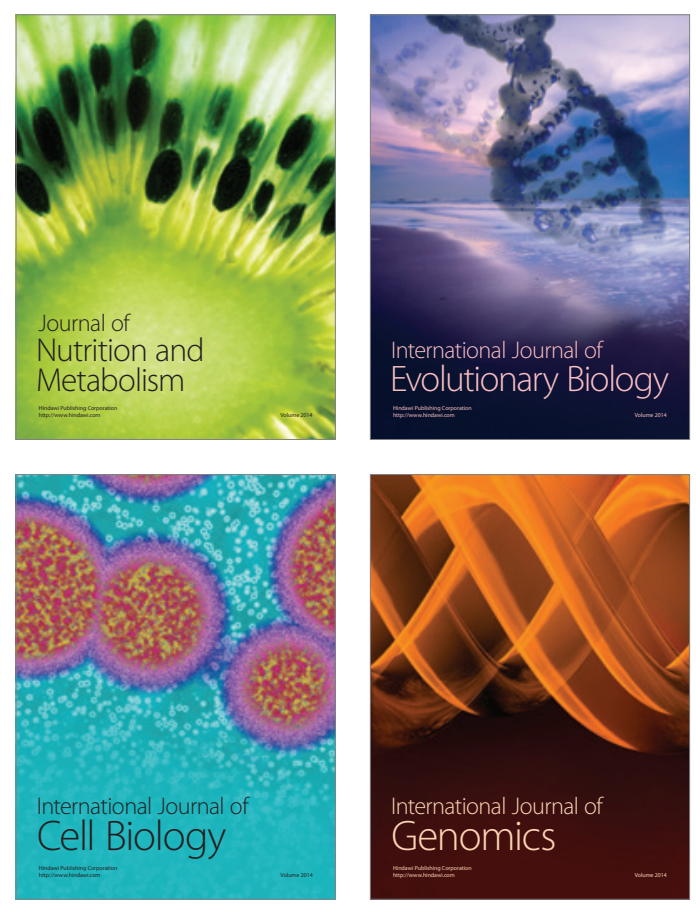
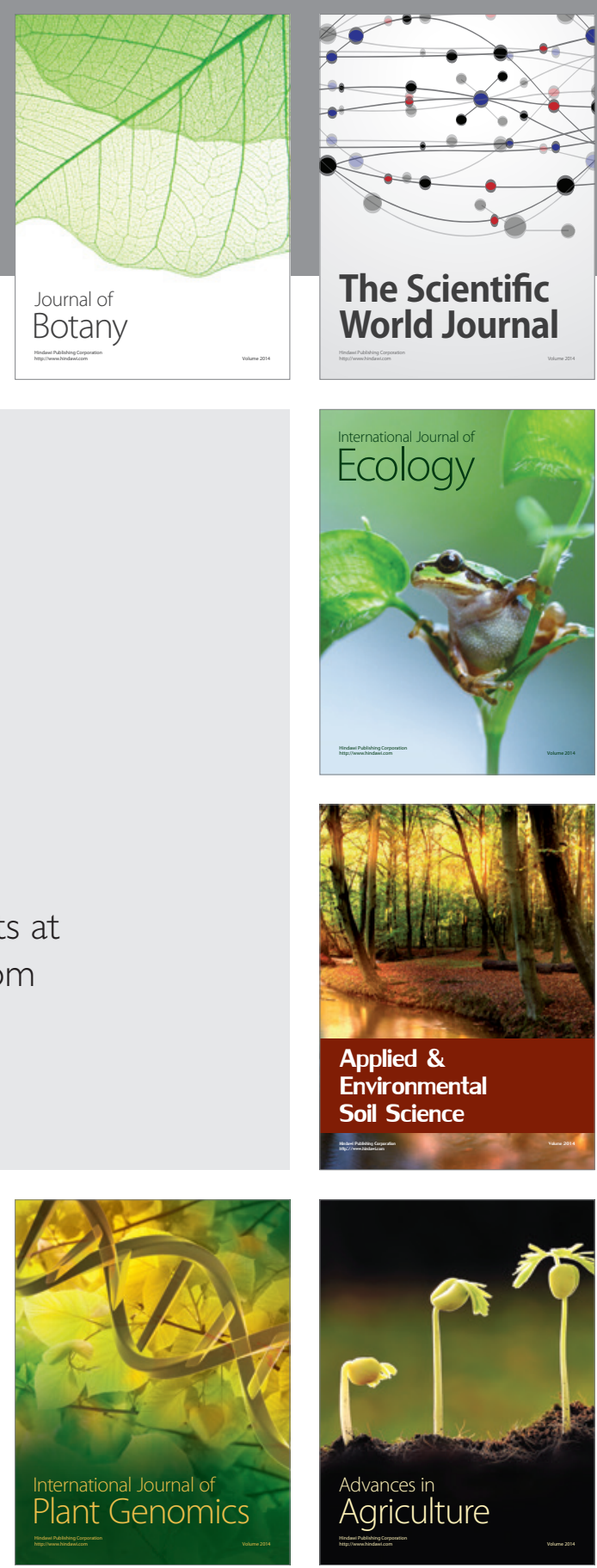

The Scientific World Journal
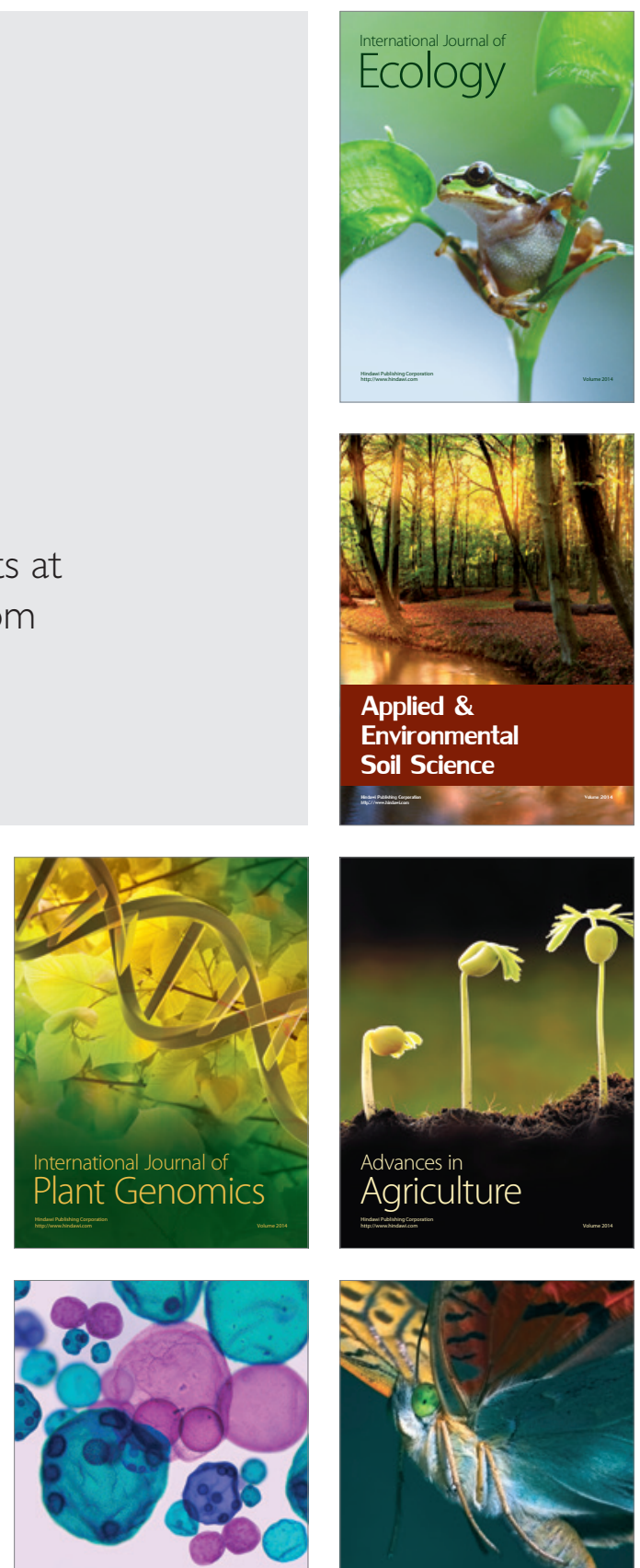

International Journal of Microbiology

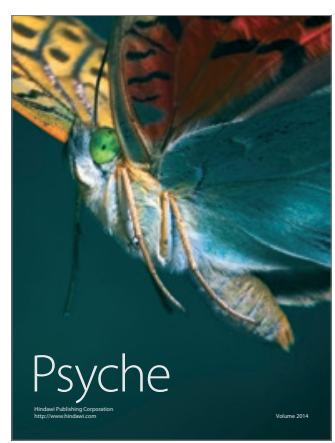

